\title{
30
}

VOLUMEIO

SUPPLEMENT I

MAY 1996

ISSN 0268-9499

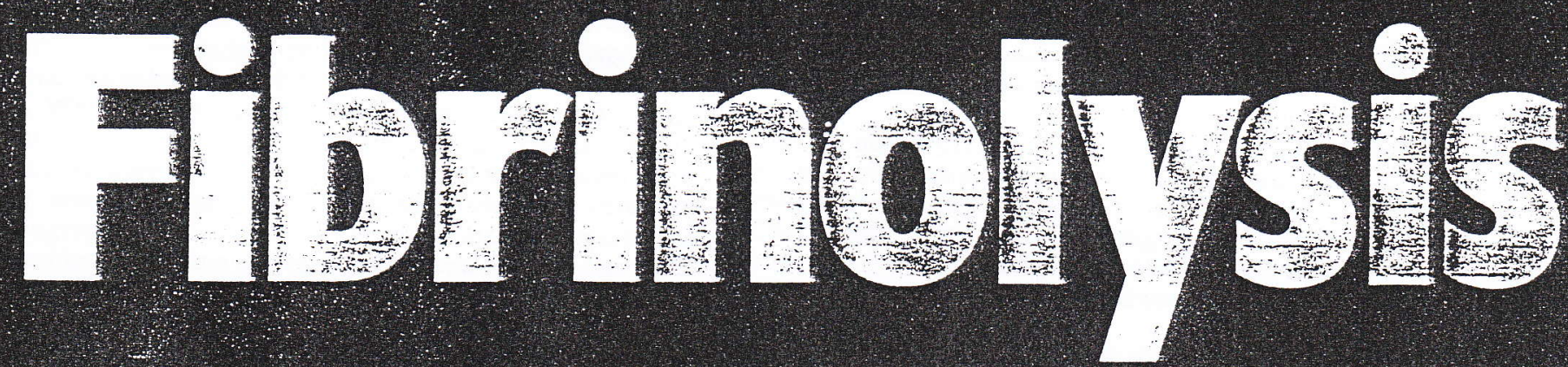

The Official lournal of the International Society for Fibrinolysis and Thrombolysis

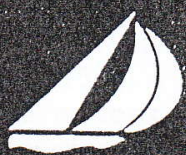

ATURARILL

LIVINGSTONE: 
182. Von Willebrand factor and prothrombin fragment $F 1+2$ in coronary and peripheral atherosclerosis

D. Stein, T. M. Schiele, M. Heins ${ }^{1}$, F.-C Schoebel, M. Sitzer ${ }^{2}$, S. Dresbach, A. Mertens, H. Steinmetz ${ }^{2}$, H. Reinauer ${ }^{1}$, M. Leschke, B. E. Strauer Medizinische Klinik und Poliklinik B, 'Institut für Klinische Chemie, ${ }^{2}$ Klinik für Neurologie; Heinrich-: Heine Universität, Düsseldorf, Germany

Increased levels of certain haemostatic factors have been shown to be related to an increased risk of cardiovascular events. Hypercoagulability is suggested to predispose to arterial thrombosis and thereby to participate in atherogenesis. We therefore assessed fibrinogen, prothrombin fragment $1+2(F 1+2)$ and von Willebrand factor (vWf) antigen in 80 consecutive patients (aged $59 \pm 5$ years) with known coronary artery disease (CAD), who all underwent coronary angiography. The extent of coronary artery disease was quantified according to modified criteria of the American Heart association (total, proximal and distal 'score'). Furthermore, the intima-media thickness (IMT) was determined in the carotid and femoral arteries by standardized ultrasonographic measurement. vWf antigen was found to correlate positively with the total and proximal coronary score $(r=0.29, p<0.05$ and $r=0.36, p<0.01)$. While $F 1+2$ showed no correlation with the coronary scores, it was significantly correlated with the IMT values in the carotid arteries $(r=0.27, p<0.05)$. After differentiating tertiles of the parameters patients belonging to the upper tertile of $F 1+2$ concentrations had significantly higher IMT values of the carotid and femoral arteries $(0.81 \pm 0.11 \mathrm{~mm}$ vs $0.72 \pm 0.13$ $\mathrm{mm}$ in the lower tertile, $\mathrm{p}<0.05 ; 1.38 \pm 0.44 \mathrm{~mm}$ vs $1.15 \pm 0.25 \mathrm{~mm}, \mathrm{p}=0.05$ ) whereas in patients belonging to the upper tertile of vWf antigen concentrations the proximal coronary artery score was significantly higher ( $1.71 \pm 0.59$ vs $1.31 \pm 0.92$ in the lower tertile, $p<0.01$ ). In this study no correlation of fibrinogen concentrations and extent of $C A D$ or IMT values of the carotid and femoral arteries could be demonstrated.

In conclusion procoagulatory mechanisms as indicated by elevated concentrations of von Willebrand factor antigen and $F 1+2$ may be contributing factors in atherogenesis.

\section{Fibrinogen and C-reactive protein are strong prognostic markers in unstable coronary artery syndromes}

H. Toss, A. Siegbahn ${ }^{1}$, L. Wallentin

Dept. of Cardiology and ${ }^{1}$ Clin. Chemistry, University Hospital, Uppsala, Sweden

The aim of this study was to investigate the prognostic importance of C-reactive protein (CRP) and fibrinogen in unstable coronary artery disease, i.e. unstable angina or nonQ-wave AMI.

Material: At inclusion P-fibrinogen and P-CRP were measured in 965 patients participating in a multicenter, prospective, placebo controlled study evaluating the effect of low molecular weight heparin in unstable coronary artery disease $(n=1506)$. Patients with newly developed or increased angina pectoris during the last two months, with latest episode of chest-pain within 72 hours, or ongoing chest-pain were included. ECG criteria to be fulfilled was transient or persisting ST-depression and/or T-wave inversion. Event rates during the 150 days follow-up period were evaluated in groups based on median levels of CRP and fibrinogen.

\begin{tabular}{|c|c|c|c|c|c|c|c|}
\hline & \multicolumn{2}{|c|}{ Results: } & \multicolumn{2}{|c|}{$\mathrm{CRP} \mathrm{mg} / \mathrm{mL}$} & \multicolumn{2}{|c|}{ Fibrinogen } & \multirow{2}{*}{$\frac{g / L}{p}$} \\
\hline & $n$ & $\begin{array}{l}6 \\
483\end{array}$ & $\begin{array}{l}\geq 6 \\
475\end{array}$ & P & $\begin{array}{l}<3.7 \\
481\end{array}$ & $\begin{array}{l}\geq 3.7 \\
479\end{array}$ & \\
\hline Day & Death & 0.3 & 3.2 & $*$ & 0.4 & 3.3 & s... \\
\hline 40 & $+\mathrm{AMI}$ & 7.0 & 9.9 & NS & 6.0 & 10.9 & $*$ \\
\hline Day & Death & 2.1 & 6.7 & A. & 2.5 & 6.3 & 4 \\
\hline 150 & $+\mathrm{AMI}$ & 11.8 & 17.1 & · & 10.8 & 18.0 & ". \\
\hline
\end{tabular}

Vaiues: \% event rate in each subgroup. "p<0.05; " $p<0.01 ; " \cdots p<0.001$

CRP strongly correlated with fibrinogen, $r=0.53(p<0.01)$. In multiple regression analysis fibrinogen tumed out to be, a strong independent marker of an increased risk.

Conclusion: Elevation of fibrinogen and CRP on admission because of unstable angina or non-Q-wave infarction indicates an increased risk of subsequent acute myocardial infarction and death.

184. Relationship between haemostatic variables and the progression of carotid atherosclerosis

E. Tremoli, D. Baldassarre, E. Cofrancesco ${ }^{1}$, C. Boschetti ${ }^{1}$, M. Cortellaro ${ }^{1}$

Institute of Pharmacological Sciences, 'Institute of Internal Medicine, University of Milan, Milan, Italy

Increases in the thickness of common carotid intima-media (CC-IMT), as measured by B-mode ultrasonography, have been widely used in both population studies and clinical trials in the search for risk factors for early atherosclerosis progression. In this study we have investigated the relations between several baseline haemostatic and conventional risk factors and CC-IMT changes over 16 months in 64 peripheral arterial disease (PAD) patients, randomiy selected from the prospective PLAT study series. Samples from 24 (37.5\%) patients who showed increases in CC-IMT during the followup period were compared with those from $40(62.5 \%)$ in which CC-IMT remained unchanged. Baseline conventional risk factors and coagulation variables were similar in the two groups except for higher plasma concentrations of von Willebrand factor (vWF) (178.3 \pm 53.6 vs $141.2 \pm 53.7 \mathrm{SD} \%$, $\mathrm{p}=0.01)$ and Factor VII (FVII) $(133.9 \pm 36.4$ vs $107.0 \pm 27.3$, $\mathrm{p}=0.001)$ in the patients with increased CC-IMT. CC-IMT increases correlated positively with plasma levels of FVII $(r=0.31, p<0.01)$ and $v W F(r=0.31, p<0.31)$. Multiple stepwise regression analysis identified FVII as the only independent variable associated with an increase in CC-IMT $(\beta=0.83$, $\mathrm{p}<0.01$ ). Thus, high plasma concentration of FVII and vWF may be associated with the progression of early carotid. atherosclerosis in $\mathrm{PAD}$ patients.

\section{Which haemostatic proteins should be used} for prognostic purposes in CVD?

\section{J. C. W. van de Loo}

Department of Internal Medicine, Münster, Germany

The study of plasmatic factors of the haemostatic system has 